\title{
Mestizaje y globalización en la arquitectura en Filipinas. Obando y Los Baños, dos casos de iglesias franciscanas de la segunda mitad del siglo XVIII/
}

\author{
Mestizaje and Globalization in the architecture \\ of the Philippines. Obando and Los Baños, \\ two samples of Franciscan churches of the second half \\ of the eighteenth century
}

Pedro Luengo Gutiérrez

Universidad de Sevilla

La presencia de los franciscanos en los alrededores de Manila tuvo una gran relevancia arquitectónica durante la segunda mitad del siglo XVIII. Recientemente se han localizado en el AFIO dos nuevos dibujos de esta fecha. Se trata de los croquis de la ermita de la Virgen de Salambao (1765) y de la iglesia de Los Baños (1782). Las constituciones franciscanas tenían especial cuidado con la arquitectura, lo que obligó a plantear soluciones concretas que afectarían más tarde a otras obras.

Palabras Clave: Virgen de Salambao; Dibujo; Convento; Pandacan; Laguna.

The Franciscan presence around Manila had a high architectonic relevance during the second half of the eighteenth century. Recently two new drawings of that time have been found in AFIO. They are plans of the Church of Virgen de Salambao (1765) and that of Los Baños (1782). The Franciscan constitutions had a special attention to architecture, therefore specific solutions were proposed, affecting later works.

Keywords: Virgin of Salambao; Drawing; Convent; Pandacan; Laguna. 


\section{Introducción}

Dentro del estudio de los fenómenos de transferencia cultural o mestizaje, el ámbito filipino ofrece una gran riqueza interpretativa. ${ }^{1}$ Esto se acentúa durante el siglo XVIII, cuando las conexiones con los puertos del Índico, del Pacífico y por ende con Europa, se hicieron más rentables, fiables y constantes. ${ }^{2}$ Para este fenómeno de mundialización, la arquitectura supone una fuente con grandes posibilidades. ${ }^{3} \mathrm{La}$ escasez de estudios recientes no se debe por tanto a la falta de interés, sino a una destacable escasez de fuentes gráficas contemporáneas. La generalización del papel de arroz como soporte para los textos y dibujos ha provocado que muchos de ellos no se hayan conservado si algún día se hicieron. Por fortuna, la catalogación tanto de los archivos estatales como de los de las órdenes religiosas está permitiendo rescatar fuentes principales para este estudio en el ámbito de la arquitectura. ${ }^{4}$ Más extraños resultan aún los referentes a obras localizadas fuera de Manila. ${ }^{5}$ La capital mantuvo una línea de comunicación con la metrópoli de la que otras ciudades filipinas carecían. Igualmente, Manila mantuvo unos modelos europeos que fuera de sus murallas no se conocían tan detalladamente. ${ }^{6}$ Por ello, los procesos de mestizaje debieron tener su origen probablemente en las provincias del archipiélago, llegando más tarde a la capital.

En este contexto, la localización de dos pequeños bosquejos de las plantas de dos edificios franciscanos del siglo XVIII ofrece un gran interés. No son simples documentos de la historia local, sino ejemplos excepcionalmente conservados de una realidad sincrética difícil de reconstruir por la

1 Gruzinski, 1993; Gruzinski, 2010.

2 Por citar algunos ejemplos, para el ámbito del Índico puede verse Quirino, 1966. Más reciente es el estudio de Martínez Shaw, publicado en Martínez Shaw y Alfonso Mola, 2000, 95-138. Para las relaciones del archipiélago con las costas americanas Yuste López, 2007. Una última referencia destacable es la ofrecida por Markovits, Pouchepadass y Subrahmanyam, 2006.

3 Un primer intento en este sentido está planteado ya en Nilsson, 1968.

4 VV.AA., 1998. También puede consultarse Elizalde López-Grueso, Fradera Barceló y Álvarez, 2001

5 Los estudios recientes de autores como Galende, Galván Guijo, Javellana o Trota José apenas aportan nuevos planos de archivo, siendo destacable su trabajo de campo. Véase Galende, 1987; Galende, 2008; Galván Guijo, 2002, Galván Guijo, 2004; Javellana, 1991, Javellana, 1997; Trota José, 1992; Trota José, 2001.

6 La presencia occidental en el resto del archipiélago fue en la mayoría de los casos testimonial, lo que fue aún más acusado en lo que a arquitectos e ingenieros se refiere. La revisión del patrimonio arquitectónico conservado fuera de Manila lleva a pensar que los modelos seguidos se conocían a través de la experiencia de la capital. 
citada escasez de fuentes. Por sus características muestra cómo las fundaciones reaprovechaban construcciones anteriores, probablemente de arquitectura civil. Además, por ello clarifica cómo la casa tradicional filipina, más conocida a partir de ejemplos del siglo XIX, estaba configurada en el siglo anterior incluso lejos de Manila. ${ }^{7}$ Por último, permite analizar el funcionamiento de la propia orden franciscana en el control de las obras de las provincias, aprovechando las tradiciones y la mano de obra local a la vez que incorporaba los modelos más habituales en la capital. ${ }^{8}$

\section{Particularidades de la arquitectura franciscana en Filipinas}

Este proceso de implantación en los diferentes territorios del archipiélago tuvo en los franciscanos a unos actores privilegiados. De hecho, estos misioneros llegaron a Filipinas en 1578, siendo en su amplísima mayoría alcantarinos. Esta línea de pensamiento de la propia orden seráfica dio un carácter particular a su presencia en las islas. A la ya de por sí reconocida importancia de la pobreza en el espíritu franciscano, se unía el carácter descalzo de esta rama. La arquitectura suponía un punto de inflexión que provocó no pocas discusiones en el seno de las comunidades franciscanas del archipiélago, más aún cuando las órdenes vecinas acometieron importantes fábricas, coincidente con el ascenso económico de las islas. ${ }^{9}$ La liturgia franciscana también implica algunas particularidades en sus conventos y de la relación en los mismos con la sociedad que se aglutinaba alrededor. Por ejemplo, legalmente los conventos nunca pertenecieron a la orden, sino al pueblo, lo que generaba no pocos problemas a la hora de administrar las limosnas para afrontar renovaciones. Quizás un ejemplo claro de esta especial vinculación entre el pueblo y los frailes es la existencia del pacaen, hoy pakaen. ${ }^{10}$ Aplicado a la arquitectura implicaría una participación directa del pueblo en la construcción de sus iglesias y conventos. Incluso las numerosas celebraciones paralitúrgicas propias de

7 Zialcita, 1980

8 Cada orden estableció unos parámetros similares en las islas, aunque todavía son poco conocidos. Hasta el momento solo agustinos, jesuitas y dominicos cuentan con trabajos concretos sobre sus fundaciones. Véase respectivamente Galende, 1987; Javellana, 1991 y Galván Guijo, 2001.

9 Cabe destacar los contenidos arquitectónicos de los estatutos de la provincia en el siglo XVIII. Luengo, 2012a, 126-128.

10 Cruickshank, 2003. 
la orden daban a sus dependencias conventuales una permeabilidad poco común. ${ }^{11}$

Otro aspecto a destacar es que el modo de poblamiento de la corona española fue radicalmente distinto al de otras potencias europeas, como ha sido puesto de manifiesto por la historiografía. ${ }^{12}$ Manila no servía solo como entrepôt puesto comercial de una red que unía el Índico con el Pacífico. Era una capital que articulaba un archipiélago, y en especial la isla de Luzón. Además, era la sede de provincias de órdenes religiosas que administraban territorios desde China hasta México. Con este contexto, Manila podría haber desistido en la ardua tarea de controlar la producción de las provincias. ${ }^{13}$ Pero por el contrario, las casas provinciales establecieron fórmulas de seguimiento de la actividad constructiva de las fundaciones, aunque no siempre de forma efectiva. ${ }^{14}$ De otra manera, no sería posible que establecimientos como Obando o Los Baños se beneficiaran de un control tan cercano desde la capital, y a la vez tan flexible. Como decían los Estatutos, debían ser misioneros enviados desde Manila los que plantearían los nuevos edificios, procedimiento que habría servido para evitar el envío masivo de planos a Manila. Esto se dejaría para casos de reformas en los planes iniciales, que debían ser consultados al provincial. ${ }^{15}$ Estos envíos muestran una cierta familiaridad de los misioneros con la representación gráfica. Las implicaciones que esto conlleva no solo afectan al ámbito arquitectónico sino también al cartográfico. ${ }^{16}$

11 El resto de órdenes no llegaron a generar normativa específica sobre estos aspectos. Quizás estos problemas no llegaron a darse, o simplemente no se consideraron destacables como para reglamentarlos.

12 Morales, 2003.

13 De hecho no se conoce que ninguna orden desarrollara esta labor en otros territorios similares como Goa con respecto a otros establecimientos en la India, por no citar las escasas fundaciones religiosas del mundo holandés.

14 «Si se hubiere de hacer de nuevo algún Convento o iglesia se ordena que [...] antes de que la obra se empieza, un religioso o dos [...] que entiendan de la materia[...] señalarán el sitio, darán la traza, y harán la planta, o mapa de dicha obra por escrito firmado de los mismos». Estatutos y Ordenanzas, 1753,36 .

15 El objeto de estas consultas no solía ser el artístico. Al contrario se buscaba controlar el gasto, el ornato y en especial la adecuación funcional de los espacios del edificio a las necesidades propias de la orden.

16 Los franciscanos, que como otras órdenes fueron responsables de gran parte de la presencia española fuera de la capital, no sólo conocían estos alejados territorios, sino que además generaron textos y aún más interesantes documentos gráficos tales como mapas o dibujos que serían fundamentales para el control desde la Provincia y el aleccionamiento de los nuevos misioneros. Luengo, 2009. Así ocurría también para las fundaciones franciscanas en el resto de América. Véase por ejemplo VV.AA., 1992. 
Como ya puede intuirse con esta reflexión acerca del control provincial desde la capital, es muy probable que Manila actuase como centro cultural de la isla de Luzón, lo que también ha llevado a gran parte de la bibliografía precedente a mostrarse especialmente atenta a lo allí ocurrido. ${ }^{17}$ Los profesionales de la arquitectura debían desplazarse desde la capital a muchas de las obras de los alrededores, lo que sin duda explica a la vez la unidad y particularidades de las iglesias filipinas. ${ }^{18}$ Las provincias de cada una de las respectivas órdenes también debían llevar un control de las obras, obligando a la remisión de informes sobre las intervenciones y enviando en no pocos casos frailes a inspeccionar las obras. Primaba la adecuación de la reforma a la regla de la orden, así como a las distintas directrices marcadas por estatutos, constituciones y otros documentos internos, básicos para la comprensión de este tipo de arquitectura religiosa, aún cuando no eran cumplidos escrupulosamente. Superadas este tipo de consideraciones, no pocos frailes se animaban a realizar críticas mucho más técnicas sobre la construcción que se llevaba a cabo en la mayoría de las ocasiones bajo dirección de sangleyes o naturales de las islas. ${ }^{19}$

En Filipinas, así como en otros territorios ultramarinos, existía la obligación de que el pueblo se responsabilizara del mantenimiento de sus propias iglesias y conventos. Por ello, existían permisos para que cortaran madera de los montes cercanos y las utilizaran en las citadas intervenciones. De todas formas, tanto franciscanos como otras órdenes, abusaron al parecer de esta excepción en distintas ocasiones..$^{20}$ En primer lugar, lo que se suponían obras de mantenimiento, se convirtieron en algunas ocasiones en verdaderas ampliaciones o reconstrucciones, lo que excedía claramente la norma. ${ }^{21}$ En segundo lugar, se debió utilizar el corte de maderas con

17 Díaz-Trechuelo Spínola, 1959; Luengo, 2008; Luengo, 2012a; Luengo, 2013.

18 Los primeros estudios referentes a iglesias de los siglos XVII al XIX ubicadas fuera de Manila son de referencia obligada, ya que muestran además una evolución en el acercamiento a las mismas. Coseteng 1972; Alarcón, 1991; Galende, 1987; Javellana 1991; Galende y Javellana, 1993; CruzAraneta y Prat 2000; Turalba c2005; Galende 2007.

19 Un caso especialmente significativo es el del agustino recoleto Lucas de Jesús María durante la construcción del Parián de Manila en la segunda mitad del siglo XVIII. Luengo, 2012a.

20 Como ejemplo se mostrarán algunos documentos de archivo que muestran esta situación, aunque existen muchos otros. En 1582, Orden del agustino Diego de Mújica para que los indios trabajen en el convento de Tondo. Archivo General de Indias (AGI), FILIPINAS, 84, N. 22. En 1694, Petición del franciscano Antonio de Santo Domingo sobre agravios a indios. AGI, FILIPINAS, 83, N. 48. En 1736, Carta de Valdés Tamón sobre cortes de madera. AGI, FILIPINAS, 384, N. 33.

21 Tablas capitulares. 1816-1862. Archivo Franciscano Íbero Oriental (AFIO) G/15. Además pueden consultarse algunos casos posteriores estudiados más detenidamente como el caso de Tondo. Luengo, 2010b. 
motivos distintos a los propuestos inicialmente, ya que también provocaron un buen número de advertencias de las autoridades. ${ }^{22}$

Este control arquitectónico desde la capital debería haberse traducido en un importante número de planos y croquis representando las distintas construcciones que se llevarían a cabo a lo largo del archipiélago, lo que en sí habría supuesto una fuente fundamental para el conocimiento de la arquitectura fuera de la capital. Pero al parecer la norma no llegó a tener efecto ya que hasta hoy no se conocían planos de conventos construidos durante la segunda mitad del siglo XVIII fuera de Manila. Solo estos dos ejemplos vienen a reabrir la cuestión de si efectivamente se llegaron a hacer y no se conservaron, o si su realización fue excepcional.

Igualmente cabría destacarlos, no solo como expresión gráfica de obras relativamente alejadas de Manila, sino como representaciones de arquitectura de este momento en Filipinas de forma general. El número total de planos conservados es verdaderamente escaso lo que puede explicarse por algunos motivos. En primer lugar el soporte, generalmente papel de arroz, cuya fragilidad ha sido la causante de que muchos documentos hayan desaparecido. En segundo lugar, y algo más interesante, el posible desinterés filipino y sangley por realizar planos. Ellos fueron en su amplísima mayoría los directores de este tipo de obras y en ninguna de las dos tradiciones existía la imperiosa necesidad de realizar un proyecto y sus correspondientes planos a la hora de levantar un edificio. Esto conllevaba un problema para los misioneros, al no encontrar delineantes adiestrados. Los alarifes sangleyes conocían las técnicas constructivas, pero no debían estar familiarizados con las técnicas de representación occidentales. ${ }^{23}$ Por ello puede pensarse que serían los mismos frailes, salvando por supuesto la labor de los ingenieros militares, los que en principio realizaron la mayor parte de mapas y croquis conservados en sus archivos..$^{24}$ Sin duda esto fue en contra de la calidad de los mismos y de una evolución de sus métodos de representación, que solo el estudio detenido de los conservados podrá valorar adecuadamente. De una u otra forma el interés reside en las especiales características de los edificios dibujados.

22 Debe recordarse la que realizara el gobernador Valdés y Tamón o más concretamente para el mundo franciscano la que se conserva en AFIO 4/3.

23 De hecho existen algunos casos de representaciones de la segunda mitad del siglo XVIII que parecen ofrecer dificultades en el uso de la perspectiva occidental. Esto puede llevar a pensar en la intervención de artistas sangleyes, que como es habitual no dejaron apenas constancia documental.

24 Luengo, 2009. 


\section{La ermita de la Virgen de Salambao (Obando)}

En el caso de Obando, se presenta en la parte superior un alzado y debajo una planta. ${ }^{25}$ De esta última pueden sacarse algunas conjeturas interesantes para comprender el devenir histórico del edificio. En primer lugar podría pensarse que este dibujo se trata de un proyecto de ermita. Llegara o no a levantarse tal y como queda dibujada, parecería indiscutible que ésta se basaría en un edificio previo, lo que explicaría tanto la forma en L, como el harigue que quedaba en medio del templo. Lo más probable es que la ermita original se correspondiera con los dos tramos verticales de la derecha. Pero si esto fuera así, implicaría la existencia de un lugar de culto en la zona previo a la aparición de la imagen, que sería ampliado en 1760. Esta posibilidad no sería excepcional en las prácticas pastorales del momento por lo que no puede descartarse totalmente.

Otra hipótesis, quizás más sólida con los datos actuales, sea que los franciscanos reutilizaron edificios civiles para la creación de una ermita. Los franciscanos, mejor que cualquier otra orden en Filipinas, podían abordar un proceso de este cariz. La casa no podía ser vendida a los frailes en ningún caso, debiendo seguir en poder de su original propietario, aunque el uso fuese estrictamente religioso. Si esto fuera cierto, la vista supondría seguramente la más antigua de una casa filipina conocida hasta el momento. Además parece claro que no se trataría de una casa exclusivamente, sino de un conglomerado típicamente urbano, que muestra además un interesante desarrollo de un asentamiento recién constituido como era Obando. Este bahay-na-bato debería corresponder con una de las casas principales del pueblo, y por tanto cercano a la plaza con la iglesia de San Pascual Bailón. ${ }^{26}$

De una u otra forma la planta es especialmente extraña. Para abordar su análisis dividiremos el edificio en dos, por un lado la planta en forma de L, mucho más espaciosa, que correspondería con el cuerpo de la ermita, y por otro el resto de patios, dependencias y otros ámbitos menos definidos. La complejidad de la segunda parte implica una diferenciación de uso en

25 Alzado y planta de la ermita de la Virgen de Salambao en Obando. AFIO 89/3

26 Con el término bahay-na-bato se conoce actualmente a la casa de piedra de arquitectura filipina. Frente a esta se encontraría el bahay-na-cubo, realizada en nipa. Se trata de un elemento patrimonial de gran fuerza en el archipiélago, por lo que su estudio a partir de fuentes históricas resulta muy enriquecedor. Hay que señalar que la construcción en piedra de la arquitectura residencial fuera de Manila y de otros centros destacados fue especialmente escasa, según el patrimonio conservado y las descripciones históricas. 
sus espacios que es difícil de recomponer. En el extremo izquierdo de la planta se observa una galería cubierta con techo raso en forma de azotea. ${ }^{27}$ En primer lugar habría que destacar la desviación del eje de su planta con respecto al cuerpo de la ermita. Sin lugar a dudas, la calidad del dibujo permite observar una cierta irregularidad en lo que con bastante probabilidad se trataba de una manzana en plena trama urbana. Este tipo de galerías bajas no son extrañas entre los bahay-na-bato conservados, pero parece no haberse terminado el piso superior. ${ }^{28}$ Entre esta galería y lo que se convertiría en iglesia, se encuentra una especie de atrio porticado, rodeado en el piso superior de balcones corridos cubiertos a dos aguas. Estas dos piezas debían haber sido planeadas como zaguán del edificio, o como organizadoras de una casa particular de gran tamaño, aunque la superposición de soluciones hace pensar en la unificación de distintas casas..$^{29}$ Tras estas dos galerías se observa claramente la existencia de un patio de dos galerías, que tampoco debía ser extraño en la arquitectura residencial con origen hispánico. De hecho cabe destacar el acceso al mismo. De una u otra forma, se trate de una agrupación de casas o no, es evidente que el patio no tenía accesos directos desde la calle, sino claramente en recodo, lo que es una característica que relaciona este edificio con obras peninsulares, donde eran ya recuerdos lejanos de la presencia islámica. ${ }^{30}$

Continuando con el alzado, cabe destacar el despiece de los sillares. Es posible que fuera una imitación realizada en pintura, pero es cierto que el acabado es similar a lo que se encuentra hoy en algunos edificios residenciales de Majayjay. Igualmente, la fachada del convento de San Juan de Dios de Manila, del que se conoce una representación gráfica del siglo XVIII, también ofrece este acabado ${ }^{31}$ En los paramentos ofrecidos por el dibujo de la ermita es claramente visible también el uso generalizado de los balcones de madera y capiz, tan propios de la arquitectura residencial fili-

27 Se entiende que el arco representado en vertical debía ser rebajado.

28 La posibilidad de que se trate de una azotea resulta extraña en esa ubicación en comparación con otros inmuebles contemporáneos conservados.

29 Cabe decir que en este punto falta correspondencia entre el alzado y la planta lo que dificulta la interpretación final.

30 Algunos investigadores han querido observar influencias islámicas en la arquitectura del archipiélago, basándose en la presencia de esta religión en las islas del sur. Resulta evidente que la posible llegada del mudéjar a Filipinas tuvo que realizarse necesariamente a través del Galeón, ya que no es una tradición cercana a lo conocido en Mindanao.

31 Maldonado de Puga, 1742. Existen varias copias de este título repartidas entre Washington, Madrid o Granada, aunque el manuscrito original se encuentra hoy en la Library of the Congress, Washington, con la signatura «Convento de San Juan de Dios (Manila)». Library of Congress (LC). Manuscript Division. MMC, 2800. 


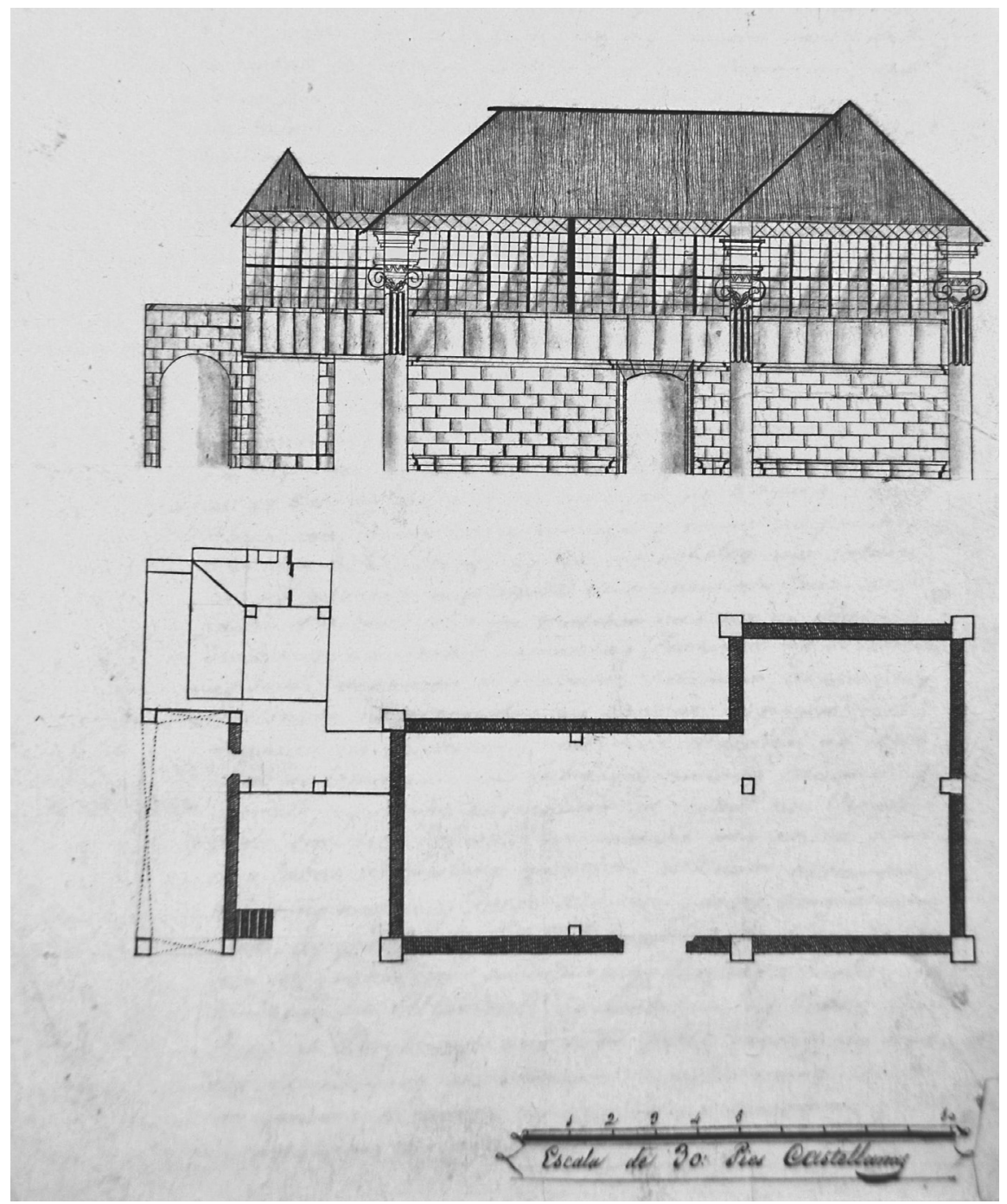

Alzado y planta de la ermita de la Virgen de Salambao en Obando. AFIO 89/3. 
pina, como excepcionales en la arquitectura religiosa. ${ }^{32}$ Sin ir más lejos, una obra característica del siglo XVIII como el Convento de San Juan de Dios no los utiliza. Incluso, habría que recordar que la orden seráfica había prohibido los balcones volados en sus construcciones a mediados del siglo XVIII. ${ }^{33}$ Un análisis detenido del dibujo muestra que el segundo cuerpo del edificio sigue los patrones de los balcones pero apenas estaban volados, lo que quizás ayudó a superar la norma. De todas formas también son conocidos algunos ejemplos poco posteriores que demuestran que esta política no se seguía estrictamente. Siendo esto así, también es cierto que los franciscanos no observaban rigurosamente sus constituciones cuando los edificios, o las obras de arte, procedían de una cesión desde otra orden, o desde particulares. Por tanto, si la ermita utilizó edificios preexistentes, tales como estos bahay-na-bato, la provincia probablemente no pondría problemas a que contaran con balcones volados o no.

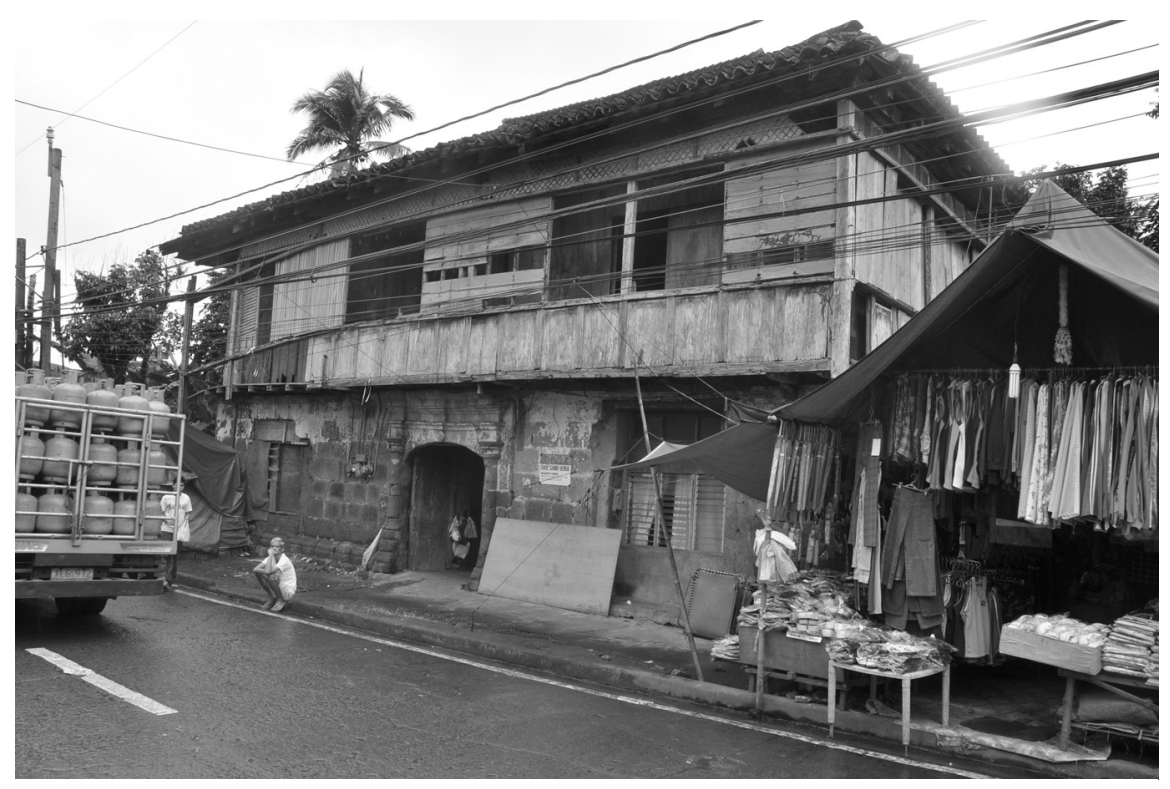

Majayjay, Laguna. Bahay-na-bato.

32 Luengo, 2012a.

33 Los Estatutos citan expresamente: «No podrán tener balcones ni ventanas voladas». Estatutos, 1753,35 . Esto apoya la posibilidad de que se trate de un edificio precedente reformado para estos nuevos usos. 
En cuanto a la decoración del balcón se sigue bastante escrupulosamente el estilo geométrico del que habla Zialcita. ${ }^{34}$ Más concretamente la ermita de Obando, así como muchos otros ejemplos conservados en el archipiélago, además de las tradicionales celosías con capiz, muestran un friso corrido con decoración de aspas o en rombos. Teniendo en cuenta que según el autor esta línea de cierta austeridad llegaría hasta la segunda mitad del siglo XIX, habría que incorporar la fecha de este edificio, construido seguramente a mediados del siglo XVIII, como comienzo de un patrón consolidado y con larga tradición posterior. En la misma línea habría que destacar el bahay-na-bato conservado hoy en Mahayhay, mucho más cercano geográficamente, y que respondería por tanto a una datación similar. $^{35}$

El techo debía ser de nipa con la inclinación característica en las casas filipinas. Más sorprendente es la incorporación de unos capiteles jónicos sobre pilastras acanaladas en su tercio superior. ${ }^{36}$ La mayoría de las casas filipinas no hacían este tipo de ostentación de unos elementos clásicos, al menos en un momento tan temprano como éste, ya que eran incluso poco comunes en las iglesias del momento. Como ya ha sido estudiado, el siglo XIX traerá consigo una revitalización de los órdenes clásicos que además de ser visibles en la arquitectura religiosa, tuvo su impronta en la residencial. ${ }^{37}$ Sin duda debía tratarse de una de las casas principales de un pueblo de muy reciente creación como era Obando. De una u otra forma implica un nivel de síntesis de tradiciones que hasta el momento era difícil de datar en muchos de los casos, y de los que no se tienen representaciones gráficas $\tan$ antiguas..$^{38}$

No cabe duda de que la propuesta para ermita en Obando parece bastante excepcional, pero es posible que estos cambios de uso de casas en

34 Zialcita, 1980.

35 Para el estudio del bahay-na-bato hay que destacar el importante trabajo que está llevando a cabo desde hace tiempo el profesor Fernando Zialcita. Zialcita, Javellana y Invernizzi Tettoni 1997.

36 El capitel jónico no deja de ser merecedor de un pequeño análisis. El cimacio parece claro en su parte superior, pero las volutas tienen un desarrollo desusado, que podría explicarse como una licencia del dibujante para conseguir así mayor detallismo. Entre ambas se observa lo que puede interpretarse como una guirnalda de flores, que no parecen colgar del ojo de la voluta, lo que probablemente respondería a modelos posteriores, sino como la decoración de la parte alta del fuste.

37 Zialcita, 1980.

38 Los dibujos de Fernando Brambila también mostrarán estas construcciones en Manila, pero algunos años más tarde. El interés del dibujo que se presenta, además de su antigüedad, es la cierta lejanía de la capital, en una zona todavía poco poblada como era Obando. 
capillas fueran bastante habituales como soluciones provisionales. Las consideraciones sobre arquitectura de los franciscanos filipinos a las que se aludió al principio de este trabajo parecen haberse olvidado en este caso, lo que no es completamente cierto, como se verá a continuación con el ejemplo de la iglesia y convento de Los Baños (Laguna).

\section{Iglesia y convento de Los Baños (Laguna)}

Las orillas de la Laguna de Bay suponen uno de los tesoros de patrimonio arquitectónico de Filipinas, de la que la orden seráfica es la principal responsable. Se trata mayoritariamente de conventos franciscanos que gozan de un aceptable estado de conservación, aunque con excepciones, por lo que es posible una fructífera comparación de los resultados constructivos de la orden en la zona. Desgraciadamente los estudios al respecto son todavía muy limitados, destacando algunos de los realizados sobre Mahayhay, Lucban, o Nagcarlan, entre otros, siendo necesaria una revisión con carácter general que ya ha sido apuntada Ahlborn. ${ }^{39}$

El primer punto que debe señalarse a la hora de tratar la presencia franciscana en Los Baños es la dificultad que los frailes tuvieron a la hora de obligar a los naturales a residir alrededor del templo. Esto ocurría aún de forma preocupante en noviembre de 1696, fecha muy tardía que conllevaba importantes contratiempos para multitud de actuaciones del gobierno español. ${ }^{40}$ La primera apuesta de los frailes por un hospital no había conseguido aglutinar a su alrededor a la población cercana. De hecho, la relación entre Los Baños y los franciscanos es mucho más conocida hoy por la ubicación del hospital de Aguas Santas que por esta iglesia de la Inmaculada Concepción. ${ }^{41}$ La presencia de los frailes en el asentamiento, ligado desde el principio al hospital, quedó interrumpida tras el incendio de 1727. Quizás el deterioro de la institución sanitaria fue vista como una posibilidad para intentar agrupar a las familias alrededor de un convento del tipo

39 Para el caso de Mahayhay véase Palazon, 1964; para Lucban Tormo y Serrano, 1971; mientras que para Nagcarlan debe citarse De Paz, 1999. Véase también con carácter general Ahlborn, 1963.

40 Mandato del oidor y visitador de La Laguna, para que todos los tributantes del pueblo de Los Baños formen y construyan sus casas en el pueblo. Lilio, 25 de noviembre de 1696. AFIO 90/14. Véase también Cruickshank, 2003.

41 Para un estudio más general sobre el hospital en Filipinas durante la presencia española cabe destacar entre otros trabajos. Casero Nieto, 1982 y Casero Nieto, 2004. 
del que había en Laguna, sin dependencias hospitalarias, que no serían recuperadas hasta $1880 .{ }^{42}$

Huerta también da algunos datos sobre el proceso de construcción y características de este edificio. ${ }^{43}$ Quizás el más interesante sea el dato de la presunta dirección de obras, atribuida por el cronista a fray Domingo Mateo. ${ }^{44} \mathrm{Su}$ biografía demuestra un ejemplo más de que los frailes, como encomendados a la administración del pueblo en sí, eran también los responsables de las obras ante la provincia, pero no sus directores arquitectónicos, caso que ha confundido algunos estudios. Las obras, una vez eran aceptadas por medio de estos informes provinciales eran subastadas públicamente a cuadrillas normalmente de sangleyes y filipinos. ${ }^{45}$ Solo el diseño de los espacios, a modo de plan general de las obras, debió estar planteado por un miembro de la orden como es este caso, y como plantean los Estatutos. A todo esto hay que añadir los promotores, quien suelen ser obviados en las crónicas. Por ejemplo, Huerta no cita el importante papel que jugó el entonces gobernador en el comienzo de la obra de Los Baños. El gobernador José de Basco y Vargas Valderrama y Rivera había nacido en Ronda (Málaga) en 1733, haciéndose cargo de la administración del archipiélago en julio de 1778. Reconocido como uno de los gobernadores que más apostaron en la modernización del archipiélago, no ha sido posible entablar una relación más estrecha bien con los franciscanos, bien con Pandacan, de la que se hablará más adelante, o con Los Baños. La documentación tampoco muestra mayor relación entre el impulsor de la econo-

42 «En el pueblo de Maynit, ha habido desde la antigüedad unos ojos de aguas medicinales sulfúreas, y para el beneficio de los baños, o vapores de ellas ha sido muy antiguo el hospital llamado de los Baños, y muy celebrado por su Fábrica sumptuosa, y de singulares providencias, y por el esmero de la Religión Seráphica en sus promptas asistencias. Hoy está llorando su ruina, por un casual incendio del año de 1726, cuyo reparo no ha podido suportar esta Real Caja, aunque corre a su cuenta la manutención posible de un beneficio tan necesario, como común». Valdés y Tamón 1739.

43 «Desde el año de 1613, hasta el de 1727 no tuvo Iglesia propia este pueblo, sirviéndole la de nuestro hospital ya mencionado[...] Mas a consecuencia de haberse quemado en dicho año el Hospital y su Iglesia, les fue preciso construir otra de caña y nipa, bajo la advocación de la misma Señora, la cual fue también presa de las llamas. La que hoy existe es de piedra, construida por los años de 1790 bajo la dirección del Reverendo Padre Fray Domingo Mateo, pero quedó techada de nipa hasta que en 1851 renovó todo el maderamen, y la techó de teja el Reverendo Padre Fray Manuel Amat[...] La casa parroquial es también de piedra y bastante cómoda, reparada y aseada en 1852 por el ya citado padre Amat», Huerta 1855.

44 Domingo Mateo, también conocido como Mateu, había nacido en San Felipe de Játiva en 1754, llegando a Filipinas en 1779, y falleció en Santa Cruz en 1797. No ostentó cargos de gran relevancia en la provincia de San Gregorio y pasó su vida entre los conventos de Pantabangan, Dilao y finalmente desde 1790 hasta poco antes de su muerte en Los Baños. Cruikshank, 2003.

45 Luengo, 2010b. 
mía filipina y los franciscanos. Lo cierto es que la iglesia se levantó gracias a su impulso, y a su donativo de 500 pesos, que parecían suficientes para la obra ya que no se vuelve a tratar la suma económica, aunque parecen escasos para afrontar una construcción así.

En este caso, los responsables de realizar el informe y el croquis de la iglesia encargados por la provincia de San Gregorio en 1782 fueron fray Alonso de Fentanes y fray José Izquierdo. ${ }^{46}$ Fentanes estaba a cargo de la iglesia de Tanay (Rizal), donde había llegado en 1768, para dejarla seguramente sin interrupción en 1784 o 1785, cuando pasaría a Manila como ministro provincial. ${ }^{47}$ Izquierdo, por su parte, había estado a cargo de Pangil (Laguna) entre 1780 y 1781, siendo en 1783 comisario visitador, cargo que ya ostentaría seguramente cuando realizó este documento. ${ }^{48}$ Los dos frailes estaban encargados de seleccionar el solar donde se levantaría el edificio. Seguramente para no interrumpir o dificultar la administración del pueblo, decidieron levantar la iglesia junto al antiguo templo en vez de reconstruirlo.

La orientación actual de la iglesia de Los Baños, aspecto que probablemente no haya cambiado con las reformas y que por tanto enlaza con la obra de Fentanes e Izquierdo, es sur. Aunque este aspecto de la arquitectura filipina merece un estudio monográfico, sí es cierto que otras iglesias del entorno como la de Morong (Rizal), la de Tanay (Rizal) o la de Binangonan (Rizal) tienen orientación norte. Ninguna de ellas debía contar con un importante caserío alrededor que justificara una orientación determinada, por lo que será oportuno observar en distintos estudios posibles razones para esta situación. Lo cierto es que las constituciones no decían nada al respecto, y quizás haya que buscar las respuestas en las prácticas geománticas sangleyes, seguramente los constructores de los edificios, antes que en razones de índole católico. Aún así no se puede negar la posibilidad de que su orientación se deba a la propia Laguna, de forma

46 Informe y croquis para construir la iglesia de este pueblo, para la cual dio el gobernador general Vasco de Vargas 500 pesos. Firma Fray Rosendo de la Transfiguración. Morong, 11 de julio de 1782. AFIO $90 / 16$

47 Alonso de Fentanes, a veces transcrito como Hentanes, nació en 1721 en Caldas (Galicia), llegando al archipiélago en 1747, y falleciendo en Maoban en 1788. Llegaría a comisario de la Orden Tercera, definidor, comisario visitador y a ministro provincial. Cruikshank, 2003.

48 José Izquierdo había nacido en Granada en 1726, llegando a Filipinas en 1747, al parecer como subdiácono. Moriría en 1791 en Santa Cruz, habiendo ostentado cargos como el de ministro de novicios, comisario visitador, definidor, viviendo en conventos de la importancia de Majayjay o Pililla. Cruikshank, 2003. 
MESTIZAJE Y GLOBALIZACIÓN EN LA ARQUITECTURA EN FILIPINAS

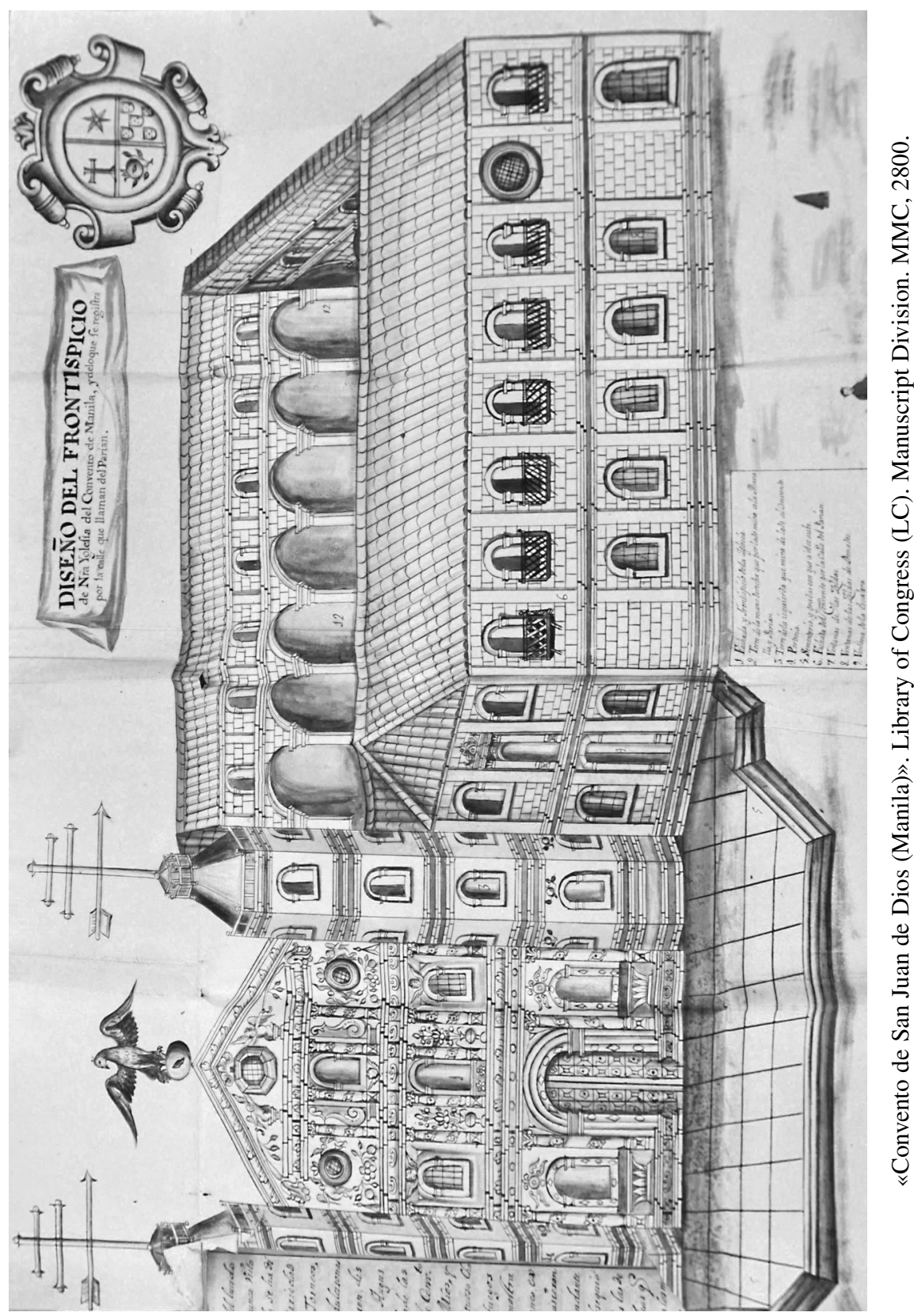


que la fachada del templo sirviera de hito arquitectónico de cada pueblo según se llegaba en barco. De todas formas el problema de la orientación va más allá de la propia Laguna de Bay como muestran ejemplos más cercanos ya a Manila, como Santa Cruz y Santa Ana de Sapa que tienen orientación sur, o Quiapo, norte. ${ }^{49}$

Además de todo esto, el dibujo requiere de un cierto detenimiento en lo que a expresión gráfica se refiere. Teniendo en cuenta la fecha del mismo, el croquis cuenta con importantes fallos de representación que podrían llevar incluso a equívoco en su interpretación. Son evidentes los problemas con las paralelas, y más aún al dibujar los muros en unos casos con dos líneas y en otros con una sola. No está realizado a escala, lo que ya era una práctica más que común en Filipinas en ese momento. La parte correspondiente a las cocinas apenas está esbozada, encontrando también una mayor preocupación en el cuerpo de la iglesia que en las dependencias conventuales. Todo esto hace pensar que el autor no estaba acostumbrado a enfrentarse a la representación gráfica arquitectónica como sí lo estaban otros profesionales contemporáneos en Manila. Sin duda se trata de un croquis más interesado en trasladar a la provincia una idea de su construcción más que un plano profesional que sirviera de base para levantar el edificio, como se deja entrever en algunos pasajes del informe. De todas formas estos errores pueden vincularse tanto a un misionero poco avezado como a un sangley poco acostumbrado a estas prácticas representativas. Por último, el plano incorpora algunas anotaciones que detallan más concretamente el proyecto constructivo..$^{50} \mathrm{El}$ informe de Los Baños (Laguna) se refiere a localización de dependencias, a ubicación de accesos y al tamaño de distintos espacios, pero no entra en otros detalles que podrían ser del mismo interés. No hay referencias técnicas, ni soluciones sobre cubiertas, ni siquiera alusiones a luminosidad, ventilación, cimentación, etc. Los aspectos subrayados por los informantes serían en cierta medida obvios para cualquier franciscano que intentaran levantar un convento en Filipinas en ese momento. Por tanto éste puede ser un ejemplo que explique también porque el proceso de control desde Manila no fue estricto.

49 Aún así, Malate y Binondo muestran una orientación este muy clara.

50 Una vara y media parece bastante de crucero, esto es, que salga fuera de la pared del cuerpo de la iglesia. Presbiterio que puede tener seis brazas a ocho, y cinco de largo con el arco toral o su arranque. Cuatro brazas podrá tener el crucero de pared a pared en lo claro, o interior.

Otros menos trascendentes son «Sacristía; y despensa. Su oficina», «Cocina y su despensa», «Patio interior, o como claustro» $\mathrm{y}$ «Batalán». 


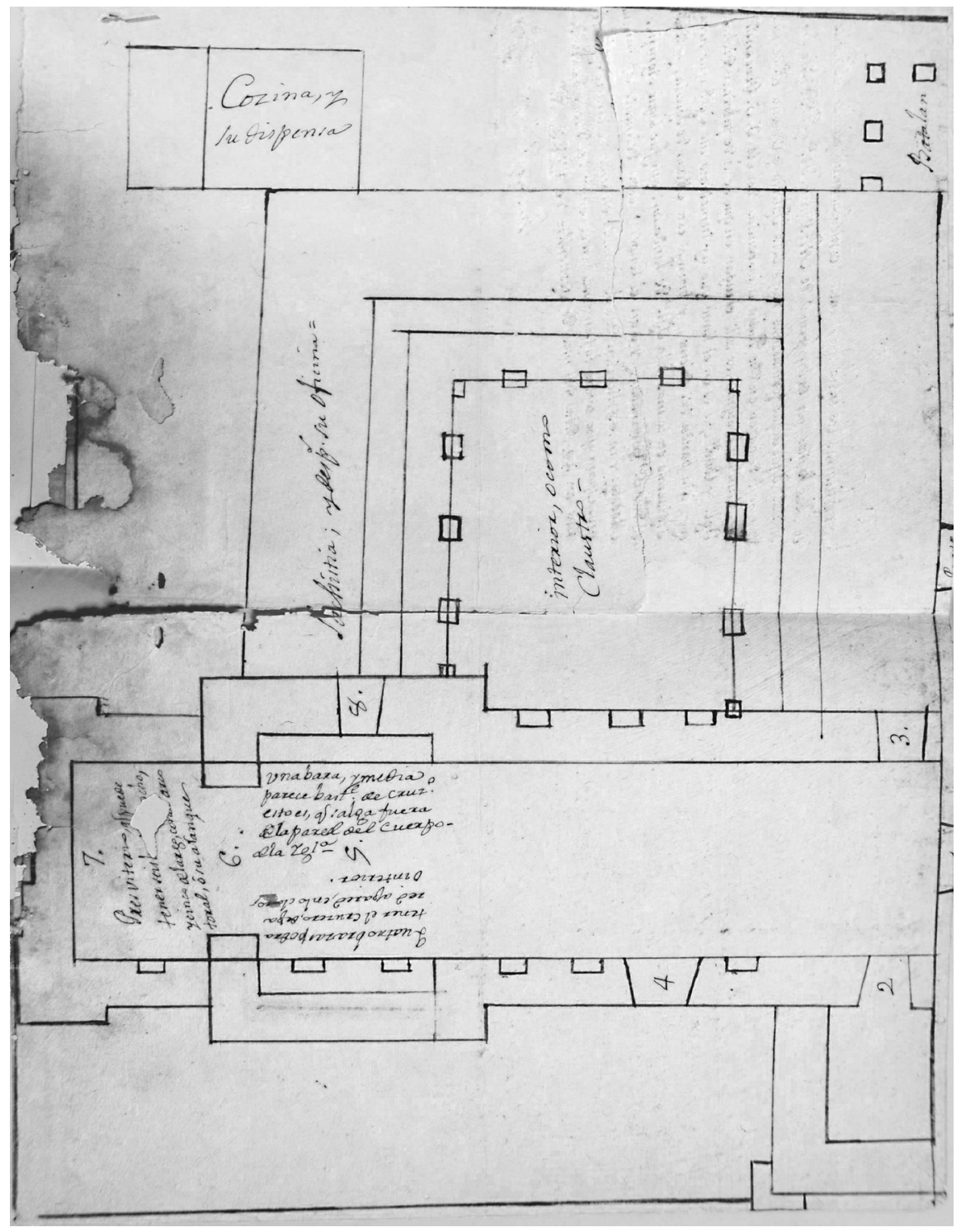

Planta de la iglesia y convento de Los Baños. AFIO 96/16. 
Antes que entrar en profundidades concretas de cada edificio se prefiere ofrecer un modelo cercano y conocido que cumpla los requisitos de la regla. Por ello, no deja de ser interesante la referencia que se hace al convento de Pandacan (Manila), como patrón a seguir a la hora de levantar Los Baños. En primer lugar hace pensar que existían unas coordenadas, más o menos definidas, que se resumían en una obra datada a principios del siglo XVIII, aunque rematada décadas antes de este proyecto. El arquetipo no era ni San Francisco de Manila, ni Santa Ana de Sapa, ni siquiera otras fundaciones franciscanas de importancia cercanas a Intramuros como podía ser Dilao (Manila), sino un convento de pequeñas dimensiones como era el de Santo Niño de Pandacan, que había sido señalado por el propio gobernador. Esto puede deberse a distintos motivos. San Francisco y Santa Ana tenían un desarrollo arquitectónico acorde con sus necesidades, muy distintas a las de los conventos de las provincias. Aunque todos se regían por las mismas constituciones, estos requerían de un tamaño en sus iglesias y conventos, que sin duda no podían servir de modelo concreto para reproducirlo. Ambas obras además respondían a criterios de principios del siglo XVIII, que podrían haber cambiado ligeramente a lo largo de las décadas, resueltos en una obra cercana cronológicamente como era Pandacan. Por ello será necesario tratar someramente las características de esta construcción. El convento fue comenzado en 1732 con el empuje del franciscano Francisco del Rosario, si bien no fue hasta 1760 cuando quedó definitivamente concluido, bajo la administración de Florencio de San José. Quedó destruido en los terremotos de 1852, siendo reconstruido completamente en varias ocasiones. Desgraciadamente no se conservan documentos gráficos del aspecto del mismo hasta el siglo XX, pudiendo utilizarse el plano de Los Baños para tener alguna noticia de su disposición.

La iglesia de Los Baños, tal y como se presenta en el plano localizado, sería de planta de cruz latina, con unos brazos muy cortos. Tendría un total de casi cuarenta y dos metros de largo por entre diez y trece - veinticinco brazas por seis u ocho-. Las capillas del crucero cubrirían un espacio de seis metros y medio por dos y medio aproximadamente - una braza y media por cuatro- . El presbiterio tenía una forma casi cuadrada, con unos diez metros por algo más de ocho, seis brazas por cinco. Con estas medidas, habida cuenta de las últimas investigaciones sobre arquitectura filipina, es necesario observar tales proporciones, para compararlas con otras edificaciones levantadas en el cinturón de arrabales de Manila en el 
siglo XVIII. ${ }^{51}$ La longitud cuadriplica el ancho, repitiendo además casi exactamente las medidas de la iglesia de Quiapo en esa misma época. Al igual que ocurría en San Francisco de Manila, y en otras muchas iglesias franciscanas de este momento, las paredes incorporaban un buen número de ventanas que ventilaban e iluminaban los templos de forma muy distinta a la de sus predecesores del siglo anterior. De todas formas, esta nueva práctica no se circunscribe a la labor franciscana ya que maestros de obras tan importantes como el sangley Domingo de la Cruz en este mismo momento plantea soluciones similares para edificios como el convento de Santa Rosa de Lima, que nunca llegó a ser construido..$^{52}$

El claustro propone una disposición bastante convencional, con las dependencias de un lado dedicadas a la portería, y frente a esta la sacristía. Como no podía ser de otra manera, ambas galerías conectaban directamente con el interior de la iglesia, la primera a través del primer tramo, y la segunda por el crucero. En paralelo a la nave del templo se encuentra una crujía donde puede que se encontrara la sala capitular, aunque también cabe la posibilidad de que se suprimiera por el escaso número de frailes que solían vivir en estas comunidades. En esta zona era donde tradicionalmente se encontraba además el refectorio y con él el acceso a las cocinas, como muestra el dibujo. La configuración del claustro era la tradicional en Filipinas, articulado probablemente por arcos sustentados por cinco pilares. En otros ejemplos franciscanos, el segundo cuerpo incorporaba ventanas de capiz que protegían del calor tropical, protegían de la lluvia y el viento a la vez que tamizaban la luz. Toda esta zona no ha resistido hasta hoy, y solo la iglesia permanece en su ubicación aunque ha sido reconstruida por completo.

Según se afirma en el mismo documento, el baptisterio debía situarse anexo al primer tramo de la iglesia, debajo de la torre. Esta disposición, $a$ priori quizás algo extraña, se conserva aún hoy en un edificio tan conocido como el de la iglesia de San Gregorio Magno en Majayjay (Laguna), y en un ejemplo mucho menos valorado como es la iglesia de Longos (Laguna). ${ }^{53} \mathrm{Si}$ bien es cierto que en aquel caso se encuentra en el lado de la Epístola, y en el de Los Baños y Longos en el del Evangelio, parece claro que se construía en el lado contrario al que se hacía el resto del convento.

51 El primer autor que valoró este tipo de informaciones para el caso filipino fue Galván Guijo, 2003. Véase también Luengo, 2010b.

52 Díaz-Trechuelo Spínola, 1959.

53 Palazón, 1964. 
Este se ubicaría en uno u otro, según las condiciones del terreno. Continuando con el tema de las torres, aquí es indiscutible que su planta era cuadrada, aunque en su desarrollo pudo ser octogonal, una fórmula fácilmente localizable en Filipinas, o bien continuar con planta cuadrada como ocurre en la citada de Longos.

En relación con las dependencias conventuales debe destacarse la existencia de un batalán porticado. Esta voz tagala fue incorporada al vocabulario castellano referido a construcción en Filipinas al menos a partir del siglo XVIII. Según vocabularios de autores dieciochescos como Noceda, en su versión de 1860 , batalán se refería a azotea de caña. ${ }^{54}$ De todas formas esta definición no coincide con lo que se desprende de otras descripciones. Más bien indica una especie de recibidor, normalmente porticado, entre las huertas y las dependencias conventuales. Este tipo de piezas son propias de los conventos filipinos y no se encuentran de forma tan generalizada en otros conventos novohispanos, lo que supone una nueva aportación de la tradición indígena filipina a la arquitectura realizada en el archipiélago en estos momentos. ${ }^{55}$

\section{Conclusiones}

A raíz de lo comentado podría pensarse que los franciscanos adoptaron un modelo poco flexible para la construcción, o reconstrucción de iglesias y conventos en las islas. Nada más lejos de la realidad. Es evidente que la provincia marcaba unas coordenadas necesarias que se cumplían en un modelo más o menos preestablecido como sería el de Pandacan o el de Los Baños. Pero también es claro que existen ejemplos aceptados por la misma provincia que plantean otros horizontes, como es el caso de la ermita de Nuestra Señora de Salambao en Obando (Bulacan). Dentro de esta flexibilidad es más fácil comprender la arquitectura en Filipinas desde la segunda mitad del siglo XVII, donde los recién llegados supieron aprovecharse del conocimiento del terreno y sus materiales no solo de los nativos filipinos, sino también de los propios sangleyes..$^{56} \mathrm{La}$ casi total inexistencia de profesionales europeos en el campo de la construcción hasta bien entrado

54 Noceda y Sanlúcar, 1753.

55 El problema es interesante si se pone en relación con lo que ocurre con elementos arquitectónicos similares en el ámbito europeo en Asia como se observa en Luengo, 2012b.

56 Zialcita, 2005. 
el siglo XVIII no hizo más que consolidar en Filipinas un modelo arquitectónico que compartía por igual tradiciones especialmente alejadas, y que supuso en cierta medida la base de autores del siglo XIX para valorar algunos aspectos de esta genuina arquitectura.

Con todo esto, se ofrecen nuevos aportes sobre la producción arquitectónica en el interior de Filipinas durante la presencia española. Son casos de integración de tradiciones artísticas muy diversas, que posteriormente tenían una sugerente difusión, primero a la capital, Manila, y posteriormente a otros territorios, hispanos o no. Además de servir de base para futuras medidas de puesta en valor de los restos patrimoniales conservados, se trata de un claro fenómeno de mestizaje arquitectónico, fruto de la transferencia cultural en un momento de madura mundialización ibérica.

Recibido el 7 de marzo de 2013 Aceptado el 6 de febrero de 2014

\section{Bibliografía}

Ahlborn, Richard: «Spanish churches of Central Luzon: The Provinces near Manila», Philippine Studies, 1963, 283-300.

Alarcón, Norma I.: Philippine architecture during the pre-Spanish and the Spanish periods, Manila, University of Santo Tomas, 1991.

Casero Nieto, Juan Antonio: «Balneario Hospital franciscano de "Los Baños" (Filipinas)», Archivo Ibero-Americano, año 42, 165-168, 1982, 850-879.

Casero Nieto, Juan Antonio: «Hospitales franciscanos en Filipinas durante la dominación española (1578-1898)», en Peláez del Rosal, Manuel (coord.), El franciscanismo en Andalucía. Conferencias del IX Curso de Verano Los Capuchinos y la Divina Pastora, Priego de Córdoba, 2004, 195-204.

Coseteng, Alicia M. L.: Spanish churches of the Philippines, Manila, UNESCO, 1972.

Cruikshank, Bruce: Spanish Franciscans in the Colonial Philippines, 1578-1898. Catalogs and Analysis for a History of Filipinos in Franciscan Parishes, Hastings, Nebraska, Cornhusker Press, 2003.

Cruz-Araneta, Gemma y Prat, Thierry: Stones of Faith. Roman Catholic Churches in the Philippines, Paris-Manila, Technology Corporation, 2000.

De Paz, Ernesto L.: Nagcarlan in perspective, Manila, Filipinas, De la Salle University Press, 1999.

Díaz-Trechuelo Spínola, M. ${ }^{a}$ Lourdes: Arquitectura española en Filipinas. 15711800, Sevilla, EEHA, 1959. 
Elizalde López-Grueso, M. ${ }^{a}$ Dolores; Fradera Barceló, Josep María y Álvarez, Luis Alonso: Imperios y naciones en el Pacífico, Madrid, CSIC, 2001.

Estatutos y ordenaciones de la Santa Provincia de San Gregorio de Religiosos Descalzos de la ... de San Francisco de Filipinas, Sampaloc, Convento de Nuestra Señora del Loreto, 1753.

Galende, Pedro G.: Angels in stone. Architecture of Agustinian Churches in the Philippines, Manila, G. A. Formoso Publishing, 1987.

Galende, Pedro G.: Philippine Church Façades. Manila, Filipiniana.net-San Agustín Museum, 2007.

Galende, Pedro G., y Javellana, René B.: Great Churches of the Philippines, Bookmark, 1993.

Galván, Javier: «Arquitectura fil-hispánica en el valle del río Cagayán», en Elizalde López-Grueso, M. ${ }^{a}$ Dolores; Fradera Barceló, Josep María y Álvarez Luis Alonso, Imperios y naciones en el Pacífico, Madrid, CSIC, 2001, 293-311.

Galván, Javier (coord.): Endangered. First international congress on hispanic architecture. Manila, Instituto Cervantes, 2002.

Galván, Javier: «Variables características de la arquitectura filhispana», en Morales Martínez, Alfredo J. (coord.), Filipinas, puerta de Oriente. De Legazpi a Malaspina, SEACEX, 2003, 83-94.

Galván, Javier: Arquitectura y urbanismo de origen español en el Pacífico occidental, Tesis inédita, Universidad Politécnica de Madrid, Escuela Técnica Superior de Arquitectura, Departamento de Ideación gráfica arquitectónica. Madrid, 2004, 2 tomos.

Gruzinski, Serge: La colonización de lo imaginario: sociedades indígenas y occidentalización en el México español: siglos XVI-XVIII, México, Fondo de Cultura Económica, 1993.

Gruzinski, Serge: Las cuatro partes del mundo: historia de una mundialización, México, Fondo de Cultura Económica, 2010.

Javellana, René B.: Wood \& Stone for God's Greater Glory. Jesuit art \& architecture in the Philippines, Quezon City, Ateneo de Manila University Press, 1991.

Javellana, René B.: Fortress of Empire. Spanish Colonial Fortifications of the Philippines. 1565-1898, Nueva York, Bookmark, 1997.

Huerta, Félix de: Estado geográfico, topográfico, estadístico, histórico-religioso de la Santa y Apostólica Provincia de San Gregorio Magno. Manila, Imprenta de los Amigos del País, 1855.

Javellana, René B.: Wood \& Stone for God s greater glory. Jesuit art \& architecture in the Philippines, Quezon City, Ateneo de Manila University Press, 1991.

Luengo, Pedro: Arquitectura conventual en Manila, 1571-1645, Sevilla, manuscrito inédito, 2008. 
Luengo, Pedro: «Los franciscanos y la representación del territorio en Filipinas, siglos XVI al XIX», Anales del Museo de América, 19, 2011, 122-139.

Luengo, Pedro: «Notas sobre arquitectura y retablos en las iglesias de los arrabales de Manila en 1782», en el III Congreso de la Feiap, Zaragoza, FEIAP, 2010a, 265-278.

Luengo, Pedro: «Notas sobre obras en la iglesia de Tondo en el siglo XVIII», Laboratorio de Arte, 22, 2010b, 217-233.

Luengo, Pedro: Intramuros: arquitectura en Manila, 1739-1762, Madrid, Fundación Española de Universidades, 2012a.

Luengo, Pedro: «Villas de recreo en los puertos europeos de Asia a mediados del siglo XVIII», Laboratorio de Arte, 24, 2012b, 377-391.

Luengo, Pedro: Manila, plaza fuerte. Ingenieros militares entre Europa, América y Asia, Madrid, Ministerio de Defensa-CSIC, 2013.

Maldonado de Puga, Juan Manuel: Religiosa hospitalidad por los hijos de... San Juan de Dios... de las Islas Filipinas, Granada, José de la Puerta, 1742.

Markovitz, Claude; Pouchepass, Jacques y Subrahmanyam, Sanjay: Society and circulation: mobile people and itinerant cultures in South Asia, 1750-1950, Londres, Anthem, 2006.

Martínez Shaw, Carlos y Alfonso Mola, Marina (dir): El Galeón de Manila, Sevilla, MECD-Focus Abengoa, 2000.

Morales, Alfredo J. (coord.): Filipinas, puerta de Oriente. De Legazpi a Malaspina, Madrid, SEACEX, 2003.

Nilsson, Sten: European Architecture in India, 1750-1850, Londres, Faber, 1968.

Noceda, Juan de, y Sanlúcar, Pedro de: Vocabulario de la lengua tagala, edición de 1860, Manila, 1753.

Palazon, Juan: Majayjay: how a town came into being, Historical Conservation Society, 1964.

Regalado Trota, José: Simbahan: Church art in colonial Philippines. 1565-1898, Manila, Ayala Foundation, 1992.

Regalado Trota, José: Visita Iglesia Bohol: a guide to historic churches, Manila, National Commission for Culture and Arts, 2001.

Roces, Alejandro R.: «Obando s Orational Dance» en Roces, Alejandro R., Fiesta, Philippines, Vera-Reyes, Inc., 1980, 75-87.

Quiason, Serafin: English «Country Trade» with the Philippines, 1644-1765, Quezon City, University of the Philippines Press, 1966.

Tiongson, Nicanor G., et al.: Igkas Arte. The Philippine Arts during the Spanish Period, Manila, CCP-AECI, 1991.

Tormo, Leandro y Serrano, Antonio: Lucban: a town the Franciscan built, Bookmark, 1971.

Turalba, Maria Cristina V.: Philippine Heritage Architecture, Pasig City, Anvil Pub, c2005.

Valdés y Tamón, Fernando: Relación de plazas, Manila, 1739. 
VV.AA.: El Extremo Oriente Ibéico. Investigaciones históricas: Metodología y Estado de la Cuestión, Madrid, AECI, 1989.

VV.AA.: Los franciscanos y el Nuevo Mundo, Sevilla, Guadalquivir, 1992.

Yuste López, Carmen: Emporios transpacíficos: comerciantes mexicanos en Manila, 1710-1815, México, UNAM-IIH, 2007.

Zialcita, Fernando: Authentic though not exotic: essays on Filipino identity, Quezon City, Ateneo de Manila University Press, 2005.

Zialcita, Fernando; Javellana, René y Tettoni, Luca Invernizzi: Filipino Style, Philippines Department of Tourism, 1997.

Zialcita, Fernando y Tinio, Martin I.: Philippine Ancestral Houses (1810-1930), Quezon City, GCF Books, 1980. 


\section{Anexo documental}

\section{Documento 1. Informe y croquis para construir la iglesia de este pueblo, para la cual dio el Gobernador General Vasco de Vargas 500 pesos. Fray Rosendo de la Transfiguración. Morong, 11 de julio de 1782. AFIO 90/16}

Fray Rosendo de la Transfiguración, Ministro Provincial de esta/ Santa Provincia de San Gregorio y siervo ¿?/ A Nuestros hermanos fray Alonso de Fentanes, predicador ex definidor/ ex comisario visitador, y guardián de Nuestro convento de Tanay, y fray José / Izquierdo, predicador ex definidor, y guardián de Nuestro convento de Pangil, salud/ y paz en Nuestro Señor Jesucristo./

Por cuanto nuestras leyes disponen, que cuando se haya de / hacer alguna iglesia a fundamentis se destinen dos religiosos/ inteligentes, para que escojan sitio competente, y dispongan/ mapa o apunte informa del tamaño y modelo que preci/samente se haya de observar en dicha fábrica, y siendo necesa/rio fabricar iglesia de piedra en el pueblo de Los Baños a ins/tancias del Muy Insigne Señor Gobernador Don José Basco y Var/gas, que para dicho efecto ha dado la limosna de 500 pesos./ Por tanto:/ Por las presentes firmadas de propia mano mando/ a Vuestras Señorías, que el tiempo y precisas ocupaciones/ de sus ministerios les den lugar, pasen a Nuestro convento del/ ya expresado pueblo, y practiquen lo arriba dicho. De este/ Nuestro Convento del pueblo de Moron, y julio 11 de 1782 años

Rosendo de la Transfiguración. Ministro Provincial. (rúbrica)

//

Certificamos los infra firmantes, como habiendo pasado al pueblo/ de Los Baños, por orden formal de Nuestro Cristianísimo Hermano y Padre Ministro pro/vincial, a fin de que formásemos mapa de la iglesia y convento, que de pie/dra se ha de hacer en dicho pueblo, a instancias del Muy Insigne Señor Gobernador/ de Manila, asignamos allí el lugar en que se ha de fabricar dicha/ Iglesia y Convento que es en el llano que hay inmediato a la iglesia que ahora/ hay, a la banda del oriente, y hicimos este mapa de Iglesia, y convento/ siguiendo en lo más a la iglesia de Pandacan, que fue lo que significo/ el dicho Señor Gobernador, y aún el convento está delineado al mismo/ modelo: y la explicación de todo se halla en frente de esta certificaci/ón. Y para que conste lo firmamos en el convento del pueblo de Los Ba/ños, en 10 días del mes de septiembre de 1782, años.

Fray Alonso de Hentanes

Fray José Izquierdo (rúbricas)

//

Este es como diseño, pues Mapa en forma, por este lo sacará / el que sepa. Los números dicen los lugares que ha de tener la iglesial y su puesto que el con- 
vento no se ha de hacer hasta que esté acabada la iglesia/ y que por ahora el orden es: que se arregle al de Pandacan, parece bastantel el diseño, que se ve supra contiguo al de la iglesia. La dicha iglesia/ podrá tener veinte y cinco brazas de largo, de pared a pared, desde/ la puerta principal, a la espalda que ha de ser del Altar mayor. Y/ el ancho de la dicha iglesia podrá ser de seis brazas, de pared,/ a pared, o como determine el Venerable definitorio de esta Santa Provincia./ Números $1^{\circ}$. Puerta principal y de la fachada de la iglesia. 2. Puerta para el bautisterio, sobre el que ha de estar la torre. 3. Puerta, por la que se entre de la portería a la iglesia y en contra. 4. Puerta traviesa, o del medio de la iglesia. 5. Capilla mayor, en que a sus lados se ve ha de tener algo de crucero. 6. Sobre que ha de estar el arco toral. 7. En donde corresponde el Altar mayor. 8. Puerta para entrar a la sacristía y en contra./ 